\title{
Efficient Energy Transport in Photosynthesis: Roles of Coherence and Entanglement ${ }^{1}$
}

\author{
Apoorva D. Patel \\ Centre for High Energy Physics and Supercomputer Education and Research Centre, \\ Indian Institute of Science, Bangalore 560012, India
}

\begin{abstract}
Recently it has been discovered - contrary to expectations of physicists as well as biologists-that the energy transport during photosynthesis, from the chlorophyll pigment that captures the photon to the reaction centre where glucose is synthesised from carbon dioxide and water, is highly coherent even at ambient temperature and in the cellular environment. This process and the key molecular ingredients that it depends on are described. By looking at the process from the computer science view-point, we can study what has been optimised and how. A spatial search algorithmic model based on robust features of wave dynamics is presented.
\end{abstract}

Keywords: Light harvesting antenna, Decoherence, Spatial search, Coherent state PACS: 03.67.Ac, 03.67.Pp, 46.40.-f

At the outset, I thank the organisers of this conference, for allowing me to talk on a topic with little direct connection to the main theme of the conference. Nevertheless, photosynthesis is a wonderful phenomenon of life with crucial involvement of quantum effects, and its detailed understanding is bound to teach us how to take advantage of quantum effects in practical problems. In the following, I present a physicist's model of the problem, sufficiently simplified for a precise mathematical treatment, and the key features of its solution.

\section{THE PROBLEM}

Life is a fundamentally non-equilibrium process. Living organisms need to stay out of equilibrium in order to survive, prosper and reproduce, and that requires a continuous supply of free energy. (When living organisms come to equilibrium with their environment, we call them dead.) The primary source of this free energy is the sun. Photosynthesis is the phenomenon whereby the chlorophyll pigments of plants capture sunlight and convert that energy in to a chemical form for later use. It is the basic step of harnessing solar energy on which most living organisms depend, either directly or indirectly through a food chain.

In the first step of photosynthesis, the energy of the captured photon is used to dissociate water (in to $H^{+}$and $\mathrm{OH}^{-}$) and create charge separation across a membrane. This is essentially an electrical step, and the resultant ions subsequently drive chemical processes for the synthesis of glucose. It is established that the efficiency of this energy conversion exceeds $95 \%$ (some quote even $99 \%$ ). One can easily estimate the efficiency by counting the number of photons captured and the number of glucose molecules synthesised, since energies of the radiation and the chemical bonds are known. On the other hand, the dynamics behind the high efficiency is still a mystery to be unraveled, especially how the dissipative effects of the chaotic cellular environment are kept out. See Ref.[1] for a review.

Even though we do not know how nature arrived at the nearly perfect optimisation of the energy conversion efficiency, we can argue that biological evolution governed by Darwinian selection would have led to it. In contrast, our best solar cells with the same aim (i.e. use the solar energy to produce charge separation in a battery) have reached only 10-20\% efficiency. Thus our energy utilisation technology, particularly at the molecular level, has much to learn from the design and functioning of the photosynthetic apparatus.

\footnotetext{
${ }^{1}$ Invited talk presented at the Symposium "75 Years of Quantum Entanglement: Foundations and Information Theoretic Applications", January 2011, Kolkata, India. AIP Conference Proceedings (2011).
} 

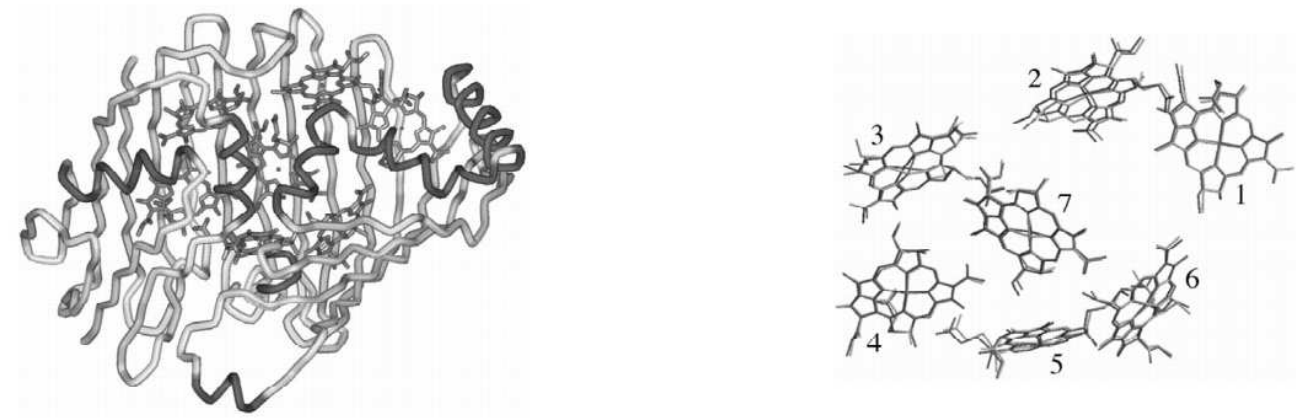

FIGURE 1. The Fenna-Matthews-Olson antenna complex, with the protein scaffold in the ribbon representation (left), and only the pigment molecules without the scaffold (right). The reaction centre is next to pigment molecule 3 . The major energy transport pathways are $6-5-7-4-3$ and $1-2-7-3$. The figure is reproduced from Ref.[2].

\section{Some Biology}

Light harvesting complexes of photosynthetic organisms display a variety, in terms of the pigment molecules involved and their structural arrangement. The variations can be understood as the organisms' adaptations to the specific living conditions, while the common features are likely to be critical to the basic function. In particular, all light harvesting complexes contain an antenna of pigment molecules that capture photons and then direct the energy to the reaction centre where glucose is synthesised. The advantages of such an arrangement are easy to understand:

- The pigment molecules are cheap, while the reaction centres are expensive. The distribution of pigment molecules in an antenna therefore increases the accessible light harvesting area at fixed cost.

- The pigment molecules differ in their sensitivity to the light spectrum and their orientations in the three dimensional antenna structure. That increases the efficiency of photon capture, given the natural variations in light direction, intensity and frequency.

- Dissociation of water requires energy of several photons of visible light that must be captured within a certain time. Light harvesting by an antenna permits accumulation of the captured energy at the reaction centre.

- The pigment molecules have a dead time after photon absorption. They also switch off when the light is too bright. These properties are easily accommodated by the antenna structure without affecting the goings on at the reaction centre.

- The pigment molecules are coupled to their neighbours via a rigid protein scaffold, and the reaction centre is next to one of the pigment molecules. That allows multiple transport pathways for the captured energy to reach the reaction centre.

As an example, the Fenna-Matthews-Olson antenna complex of the purple bacteria is shown in Fig.1. It contains 7 bacteriochlorophyll pigment molecules, with neighbouring distances around $20 \AA$. The geometry is not that of a star or a ring or some other regular graph, and the reaction centre is not in the middle. The energy transfer time between neighbouring pigment molecules is observed to be about $50 \mathrm{fs}$.

Experimental investigations of the dynamics of energy transport in the light harvesting complexes have thrown up a surprise. The process shows wavelike coherent oscillations, going back and forth and lasting longer than 400fs, which is quite distinct from classical random walk hopping between neighbouring pigment molecules. The observations were made first at liquid nitrogen temperature for purple bacteria containing the FMO complex [3], and then at ambient temperature for marine cryptophyte algae containing PC645 and PE545 antenna proteins with 8 bilin molecules [4].

The experiments are in the framework of two-dimensional spectroscopy. They have used lasers on light harvesting complexes extracted from cells, and not sunlight on living cells, but that is not believed to alter the results. First one laser of frequency $\omega_{1}$ injects energy into the complex creating an exciton, and then after a time lapse $\tau$, a second laser of frequency $\omega_{2}$ extracts the energy from the complex by stimulated emission. The results are displayed as 
intensity correlations in the $\omega_{1}-\omega_{2}$ plane, evolving as a function of $\tau$. (A video of the results of Ref.[3] is available as supplementary information to the online version of the paper.) The diagonal peaks in the plot are trivial, while the off-diagonal peaks describe couplings between different frequency modes. The results show several oscillations in the off-diagonal signal, going on without appreciable decay till the maximum value of $\tau$ explored. (The maximum value of $\tau$ was set by theoretical estimates, which turned out to be incorrect, and hence we do not really know for how long the oscillations persist.). The long-lasting coherent coupling between excitonic modes is the feature that is closely tied to efficient energy transport and that a realistic model of the process must explain.

\section{Some Chemistry}

Basic chemistry involved in photosynthesis is well-known [1]. Since the visible light wavelength is much larger than the separation between pigment molecules, several pigment molecules can capture a given incident photon. Still, every photon that falls on a light harvesting complex is not captured. When the photon is captured, it creates a state called exciton. Excitons are extended molecular (not atomic) states, often spanning two pigment molecules over nanometre distances. Various excitons in a light harvesting complex do not have the same energy. Nevertheless, coherent energy transfer occurs between them, as clearly demonstrated by the two-dimensional spectroscopy experiments.

The pigment molecules are too far away from each other for their molecular orbitals to overlap. They interact with each other through the long range dipole-dipole interaction. Photon absorption produces a polarisation cloud, without any ionisation. Coulomb forces propagate the polarisation from one pigment molecule to its neighbour. The tight covalent binding of the pigment molecules to the protein scaffold couples this propagation to vibrational modes, and the energy pulse hops around in the antenna complex.

A simple classical strategy for directing energy from the antenna of pigment molecules towards the reaction centre is to have a funnel-shaped energy landscape. That requires maintenance of permanent energy gradients which is expensive, and using one battery to charge another is certainly counterproductive. Furthermore, a non-dissipative pulse spends the least time near the potential minimum, and so needs to be trapped at the right moment to be useful. Needless to say, energy transport in photosynthesis does not follow this strategy.

A realistic strategy has to incorporate the fact that the exciton energy accumulates at the reaction centre, with a wavelike transport and without dissipation. Furthermore, the energy needs to be trapped at the reaction centre for a duration long enough ( $\sim$ few ps) for conversion to chemical form. Algorithms that can support such features have been discovered in the study of quantum computation.

\section{Some Computer Science}

A physical process evolves some initial state to some final state according to some interaction inbetween. That can be considered a computation, provided a suitable map can be constructed between physical properties of the system and abstract mathematical variables. In such conditions, different physical interactions give rise to different types of computers, while efficiency and stability of these computers depend on the nature of the physical interactions implemented.

The traditional computational paradigm implements discrete Boolean logic using digital electronic circuits. It is an instance of classical particle dynamics. Quantum computation considers unitary evolution of quantum states in a Hilbert space. That includes properties of both particle and wave dynamics, and hence is more powerful than classical computation. In a similar vein, one can look at wave computation [5], which is different from Boolean logic computation but contained in quantum computation. Analog variables in wave computation can superpose, interfere, disperse etc., but without any entanglement.

Historically, waves have been widely used in communications, but their properties have hardly been exploited in computation. Wave algorithms can cover the same Hilbert space as quantum computers, and the two have the same time and oracle complexity. The two differ, however, in their space requirements and stability properties. Explicitly, an $N$-dim Hilbert space can be realised using $N$ wave modes but only $\log _{2} N$ qubits. On the other hand, wave dynamics is much more robust against environmental disturbances than quantum dynamics is. A practical computer always needs a trade-off between minimisation of resources and minimisation of errors. Thus a wave computer can be advantageous in situations, where the problem size is modest, spatial resources are cheap and a quantum computer would be fragile. 
A change in physical implementation of a mathematical algorithm can also alter its interpretation. Both quantum and wave computers evolve amplitudes of their states, subject to conservation of the norm. The square of an amplitude, $|A|^{2}$, gives the probability of a state in quantum systems, while it gives the energy of a mode in wave systems. In particular, an amplitude amplification algorithm describes a search process in the quantum setting and an energy focusing process in the wave setting. Search algorithms and their optimisation have been extensively investigated in the framework of quantum computation. Their wave versions are efficient schemes to transfer/redistribute energy with many practical applications-from mechanical systems to chemical, electrical and biological ones.

A wave dynamics realisation of Grover's algorithm is what a mechanic intuitively does to find a structural defect in an object - give it a vigorous shake. The vibrational modes go out of phase at the location of the defect, which leads to amplitude and energy enhancement at that place and subsequent cleavage. Parameters of the process, vibrational frequencies and phase shift at the defect, can be optimised for the best results. But even when the parameters are not perfectly tuned, the algorithm works reasonably well providing useful results.

With this background, we can see that the energy transport problem in photosynthesis maps to the spatial search problem, where an initial amplitude distribution over many locations evolves by transfer between neighbours and gets concentrated at a specific target location. To ascertain whether that is indeed what nature has implemented, we need to figure out: (1) To what extent is the physical hardware of the light harvesting complex capable of supporting the spatial search algorithm? (2) Are there any experimentally testable characteristic signatures indicating the role of wave dynamics in the optimal solution to the problem?

\section{Some Physics}

Since the discovery of coherent energy transport in photosynthesis, many attempts to model it on the basis of quantum dynamics have been made (see for instance, Refs. $[6,7,8]$ and references therein). These have analysed effects of entanglement, decoherence and environmental noise in fully quantum systems. My goal here is more modest - the process can be understood using classical dynamics of waves, without invoking full-fledged quantum dynamics.

Any state in an $N$-dimensional Hilbert space can be decomposed as $|\psi\rangle=\sum_{i=1}^{N} c_{i}|i\rangle$. Its evolution is called coherent, when the relative phases of $c_{i}$ (corresponding to the off-diagonal elements of the density matrix) are protected from external disturbances. It is called entangled, when specific to a choice of subsystems, the state cannot be expressed as a product of the subsystem states. Thus coherence is a generic property arising from superposition, while entanglement always refers to division of the system in to smaller subsystems. A given Hilbert space can be realised in many ways depending on the physical components involved. When it is a direct sum of distinct modes (i.e. $N=1+1+\ldots+1$ ), it exhibits superposition without any entanglement. When it is a tensor product of smaller spaces (i.e. $N=N_{1} \times N_{2}$ ), entanglement is possible. It is the former realisation that appears in wave computation, while the latter one is routinely used in quantum computation.

In the real world, all wave processes suffer decoherence and damping due to interaction with the environment. But it is observed that decoherence is much more rapid than damping (i.e. reduction in the norm of the state), and superposition is much more stable than entanglement. These properties make classical wave algorithms much more stable against environmental disturbances than their quantum counterparts. Indeed, a variety of wave systems with coupled vibrational/rotational modes and small damping can be constructed easily.

With the above concepts in mind, we can list the physical ingredients that can produce long-lived coupled wave modes in the light harvesting antenna, as the hardware for implementing the energy transport algorithm:

- The dipole-dipole interaction leads to vibrational motion of the polarisation cloud. The charge density degree of freedom (i.e. $\rho=\psi^{*} \psi$ ) does not involve the electronic wavefunction phase that decoheres rapidly. But it does carry the more robust classical vibrational phase.

- Classical wave modes belong to the "decoherence free" subspace of the full quantum space. They correspond to coherent states, which are eigenstates of the annihilation operator and not energy eigenstates. Then, by definition, they remain unaffected by linear coupling to the environment.

- Decoherence rates are often estimated from system-environment weak scattering cross-sections, dilute gas approximation and Fermi's golden rule. Such calculations generically overestimate decoherence $[9,10]$. In particular, the estimates are invalid for frequent interactions (what is supposed to escape irreversibly actually returns), as well as for adiabatic interactions (slow phase disturbances cancel in case of cyclic evolutions). As a result, only environmental modes with evolution time scales comparable to that for the system contribute significantly 


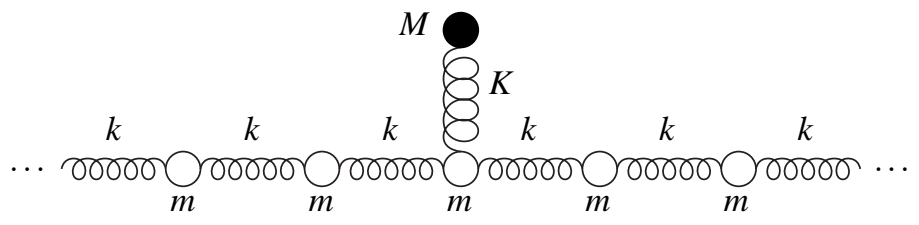

FIGURE 2. A linear chain of identical harmonic oscillators, coupled to a side branch oscillator that acts as a storage cavity.
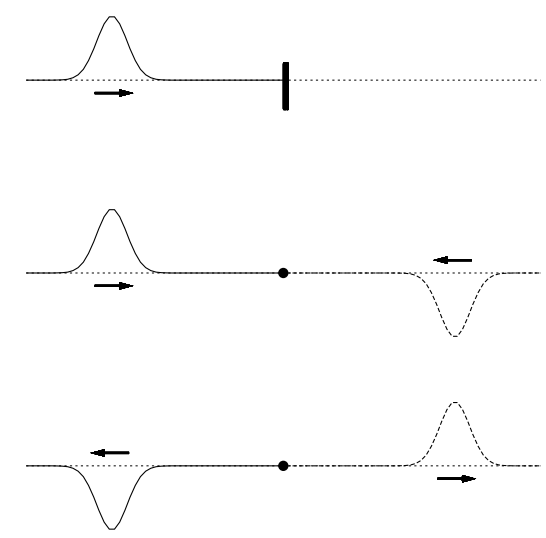

FIGURE 3. The reflection oracle for a wave pulse incident on a hard wall. The node at the wall (top) is simulated using the method of images (middle), and the linear superposition evolves to have the amplitude of the reflected pulse reversed in sign (bottom).

to decoherence of waves. In this context, it is worth noting that the photon energy being transported during photosynthesis is considerably larger than the energy of the thermal phonon disturbances.

\section{A SIMPLE MODEL}

Having described the essential requirements of an efficient energy transport algorithm, we now look at an explicit model of it involving wave computation. The spatial search algorithm [11] is a variation on Grover's database search algorithm, where locality of physical interactions limits the movement during the search process from a location to only its neighbours. Geometry and connectivity of the distributed database are important features in the process. The algorithm needs two basic operations: amplitude transfer from any location to its neighbours and an amplitude reflection oracle at the target location. The optimal solutions require database exploration with relativistic dispersion relation, which is satisfied by wave propagation but not by diffusion.

A model of coupled simple harmonic oscillators that can implement the algorithm is displayed in Fig.2. The $N$ identical oscillators representing the pigment molecules are shown as a linear chain, but they can also be arranged in a network with suitable connectivity. The reaction centre is represented as a different oscillator on a side branch. The nearest neighbour coupling among the oscillators allows amplitude transfer with relativistic dispersion relation. Reflection of a wave pulse from a hard wall flips the amplitude in sign, as depicted in Fig.3 using the method of images. For a propagating wave pulse, the connection to the side branch acts as a beam-splitter, where reflection as well as transmission occur. The frequencies of the oscillators are the parameters that have to be optimised, together with the geometry of the oscillator network.

It is important to note that in the optimisation of biological resources, as exemplified by Darwinian evolution, energy is more important than time (living organisms slow down their metabolism in adverse environments), and time is more important than space (parallel processing is commonplace). These criteria differ from those normally used in studying computational complexity. In the present case, the dynamics of the oscillators is to be optimised for the most efficient energy transfer, and not for the quickest energy transfer.

The mathematical algorithm that does this job was written down by Tulsi [12]. It works in an $(N+1)$-dimensional Hilbert space, with the extra state playing the role of a trapping cavity at the target location. The amplitude evolves 
essentially in the three-dimensional subspace of the complete Hilbert space, formed by the cavity state, the target state coupled to the cavity and an "averaged" non-target state. Clever transfer of amplitude between the distributed database and the cavity allows $O(1)$ of the initial energy to accumulate in the cavity. That amounts to a resonance condition: For constructive build up of the amplitude, the phase change for the energy pulse to cycle in the oscillator network has to match the phase change in the side branch. (Note that a similar strategy is used in gravitational wave detectors, where a weak vibrational signal accumulates in a high $-Q$ Fabry-Pérot cavity, until it crosses the detection threshold.) Indeed, the resonance condition is a prediction of the algorithm that can be experimentally tested as a requisite property of the light harvesting complex.

The experimentally studied light harvesting complexes have a small number of pigment molecules, seven for the purple bacteria and eight for the marine cryptophyte algae. In such situations, instead of looking at the asymptotic behaviour of the algorithm, one should look for specific values of $N$ and the corresponding connectivity of the oscillators that yield high energy accumulation efficiency. (Such efficient solutions are well-known for Grover's database search algorithm.) Detailed analysis and results will be presented elsewhere.

\section{ACKNOWLEDGMENTS}

I am grateful to Graham Fleming, Anthony Leggett and K.L. Sebastian for useful discussions. I thank the audience for their many comments and questions that helped me clarify the concepts presented here.

\section{REFERENCES}

1. R. van Grondelle et al., Biochimica et Biophysica Acta 1187, 1-65 (1994).

2. A. Camara-Artigas R.E. Blankenship and J.P. Allen, Photosynth. Res. 75, 49-55 (2003).

3. G. S. Engel et al., Nature 446, 782-786 (2007).

4. E. Collini et al., Nature 463, 644-647 (2010).

5. A. Patel, Int. J. Quant. Inform. 4, 815-825 (2006); Erratum ibid. 5, 437 (2007).

6. K. B. Whaley, M. Sarovar and A. Ishizaki, in "Quantum Effects in Chemistry and Biology", Proceedings of the 22nd Solvay Conference on Chemistry, e-print arXiv:1012.4059 (2010).

7. F. Caruso et al., Phys. Rev. A 81, 062346 (2010).

8. V. Vedral and T. Farrow, e-print arXiv:1006.3775 (2010).

9. A. J. Leggett, in Quantum Mechanics of Complex Systems, I and II, Proceedings of the 1989 NATO Advanced Study Institute, Evora, Portugal, Plenum Press, New York, 1990.

10. W. G. Unruh and W. H. Zurek, Phys. Rev. D 40, 1071 (1989).

11. N. Shenvi, J. Kempe and K. B. Whaley, Phys. Rev. A 67, 052307 (2003).

12. A. Tulsi, Phys. Rev. A 78, 012310 (2008). 